\title{
Method of Planes Normal Pressure for Slit Geometries in Molecular Dynamics Simulations
}

\author{
Sz. Maćkowiak ${ }^{1}$, D.M. Heyes ${ }^{2}$, D. Dini' ${ }^{2}$, A.C. Brańka ${ }^{3 *}$ \\ ${ }^{1}$ Institute of Physics \\ Poznań University of Technology \\ Nieszawska 13 a \\ 60-965 Poznań, Poland \\ ${ }^{2}$ Department of Mechanical Engineering \\ Imperial College London, Exhibition Road \\ South Kensington, London SW7 2AZ \\ United Kingdom \\ ${ }^{3}$ Institute of Molecular Physics \\ Polish Academy of Sciences \\ M. Smoluchowskiego 17 \\ 60-179 Poznań, Poland \\ *E-mail: branka@ifmpan.poznan.pl
}

Received: 13 May 2013; revised: 1 August 2013; accepted: 2 August 2013; published online: 5 September 2013

\begin{abstract}
The resolution and convergence properties of the Method of Planes (MOP) local pressure tensor method is analyzed for a slit geometry in which a system of interacting particles is placed between movable walls composed of atoms. Boundarydriven Molecular Dynamics (BMD) simulations were performed for different situations in which solid or fluid phases are formed between crystalline or amorphous walls. It is shown that for these inhomogeneous, steady state structures the total force exerted by a wall atoms on the inside particles is consistent with the normal pressure component obtained from the MOP method if a sufficiently small integration time step is applied. The work demonstrates that the numerical errors associated with computing the MOP pressure can be non-negligible and should be a consideration when determining the BMD algorithm parameters.
\end{abstract}

Key words: pressure tensor, method of planes, molecular dynamics, computer simulations, inhomogeneous systems, slit geometry

\section{INTRODUCTION}

Over the last three decades molecular dynamics methods to investigate systems of interacting particles under externally applied pressure or stress components have been developed in molecular dynamics simulation. These isobaric or isostress methods, often referred to as "barostats" are routinely used to study the physical properties of the bulk condensed matter systems [1]. In the barostat scheme scaling of the molecular coordinates is used to make strain tensor changes in accordance with the predefined stress component values.
In this way coupling of a physical system to a "pressure bath" was introduced. Different thermodynamic conditions can be realized and systems in different thermodynamic ensembles be investigated [2-4].

The first pressure control method for molecular dynamics (MD) simulation was derived by Andersen [5]. The Andersen barostat is based on an extended Hamiltonian formulation of the equations of motion for the molecules and the cell volume, $V$. The cell volume is driven by a fictitious piston. Scaling of the molecular coordinates by $V^{1 / 3}(t)$ is employed 
to achieve on average a target pressure, $P_{e x t}$ via a feedback mechanism. The equation of motion for the box volume is $\ddot{V}=\left(P(t)-P_{\text {ext }}\right) / M$, where $P$ is the instantaneous pressure of the system calculated by the virial formula and $\mathrm{M}$ is the piston "mass".

Variants on the Andersen method have been developed since [6]. There are also generalizations to achieve a predefined stress tensor component, $\boldsymbol{P},[4,8-10]$. which employ a variation of the simulation cell shape and hence strain. An alternative approach (a constraint type of method) forces the virial $P$ be held constant at a value of $P_{\text {ext }}$ and adjusts the volume, $V$, to achieve $d P / d t=0$ [11]. A weak coupling barostat was proposed by Berendsen et. al. [12] in which the volume is scaled by a factor proportional to instantaneous pressure fluctuations, $P-P_{e x t}$. All of these 'bulk system' barostats employ uniform scaling of the molecular coordinates, with the instantaneous pressure calculated using the virial formula.

There is increasing interest in liquids confined in nanosized geometries, either at equilibrium or in flow, where the gap between the walls may only be a few molecules thick. In a commonly employed geometry, periodic boundary conditions are applied in two of the cartesian directions, while the liquid is bounded by walls in the remaining direction. Such a geometry can be used to represent a liquid lubricant film or two solid walls in ('dry') contact where the walls slide with respect to each other. The distance between the walls is allowed to vary with time in response to an applied normal load or pressure. This situation is quite different and less well studied than the bulk-system analogues. The barostat requires a distinct pressure control solution which is not based on the virial pressure formula [13-17]. The walls cause inhomogeneities in the liquid density and other properties (and their fluctuations). Homogeneous scaling of the coordinates might therefore cause unphysical states to appear by virtue of a uniform coordinate scaling barostat procedure. Furthermore the equilibrium statistical mechanical form of the pressure tensor or thermodynamic route is inapplicable away from equilibrium. Spatial variations of the stress tensor in an atomistically inhomogeneous system requires a local pressure tensor definition.

There are two methods used to determine the local pressure tensor for an atomistic fluid flow in slit geometry which are valid for highly inhomogeneous, nonequilibrium fluids.

The first is based on a local volume average extension of the bulk virial pressure formula to a sub-volume or "bin" of volume [18]. The second, Method of Planes (MOP) route, is based on an average of the molecular interactions and crossing through a plane [19]. The equivalence between volume averaging (VA) and MOP definitions of the pressure tensor at a plane has been proved and demonstrated by MD simulation [20]. In the slit geometry we define $z$ to be the direction orthogonal to the wall plane.
In a strongly confined systems such as a slit geometry it is most realistic to make the walls of discrete atoms, in part because the temperature and/or pressure control is implemented directly through the wall atoms [21]. In a slit, the tensor component $P_{z z}$ (or normal pressure) must be constant throughout a stationary fluid and it can be calculated by MOP with a plane positioned at any z-value in the fluid. Furthermore it must be equal to the total z-force per unit area, exerted on the fluid particles by the wall atoms. As a result of the combined action of confinement, temperature, pressure and shear, different non-fluid arrangements of atoms can be realized, such as a stationary coexistence between a strained crystal and a sheared liquid by boundary-driven shear flow or plugslip state $[22,23]$. In these cases, the way in which the MOP method can be exploited has been less well discussed in the literature. In particular, the important role of the applied integration time step has not been fully recognized. In this work this issue is analyzed by considering the total z-force exerted by the wall atoms and results obtained from the MOP method for a model slit pore subject to different physical and numerical conditions. In Sec. II the MOP method is introduced. Simulation details and the obtained results for different parameters of a confined atomic film are discussed in Sec. III. Conclusions are presented in Sec. IV.

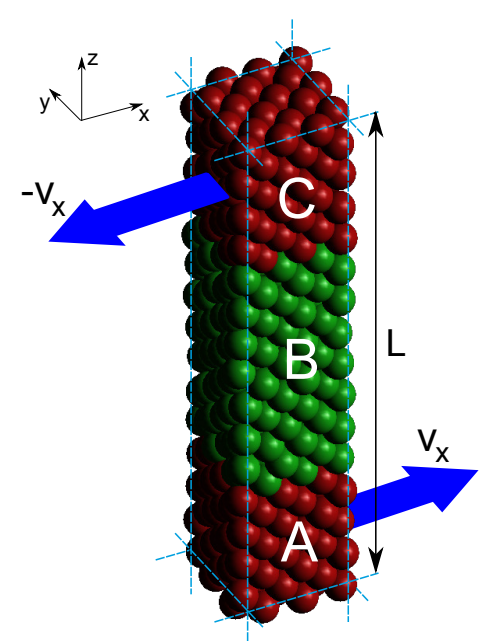

Fig. 1. A schematic diagram of a simulation slit geometry. The outer atoms in region $\mathrm{A}$ and $\mathrm{C}$ are tethered to lattice sites with harmonic springs and form the confining walls. The simulation cell is periodic in the $\mathrm{x}$ and $\mathrm{y}$ directions and the walls can translate along $\mathrm{x}$-direction

\section{THE MOP METHOD}

The geometry of a confined liquid or solid film positioned between two parallel walls (in the $x z$ plane in the diagram) is shown in Fig. 1. The atoms in the central region B undergo classical equations of motion without any externally imposed body (constraint) forces. In the two outer regions (labeled A 
and $\mathrm{C}$ in the figure), external constraints such as uniform translation or applied normal or shear force can be applied, and the atoms may be anchored or tethered to sites of an underlying rigid lattice of sites. The density of the system is defined for a fixed value of the distance $L$ between the walls.

An expression for the local pressure tensor which is applicable quite generally to inhomogeneous equilibrium or nonequilibrium systems suitable for the geometry of Fig. 1 was derived by Todd et al. [19]. They incorporated the full Irving-Kirkwood expansion for the pressure tensor at time, $t[19,25]$. The expression is formulated in terms of the force and momentum transfer across a plane and was named the Method of Planes (MOP). It is decomposed into kinetic and potential (or configurational) parts, i.e., $\boldsymbol{P}=\boldsymbol{P}^{K}+\boldsymbol{P}^{U}$ just like the bulk virial expression. The kinetic component of MOP is

$$
\begin{aligned}
& P_{z z}^{K}(z)= \\
= & \lim _{\tau \rightarrow \infty} \frac{1}{S \tau} \sum_{0<t_{i, m}<\tau} \sum_{i} p_{z i}\left(t_{i, m}\right) \operatorname{sgn}\left[p_{z i}\left(t_{i, m}\right)\right],
\end{aligned}
$$

for a time-average, where $\tau$ is the simulation time, $S$ is the cross section area, $t_{i, m}$ is the set of time events $(m=1,2, \ldots)$ in which $i$ particle crosses a plane, $p_{z i}$ is the z-component of momentum of $i$-particle, and $\operatorname{sgn}$ is the signum function. Thus, the kinetic part of the pressure tensor in MOP is the time average over a sum of the momenta of particles which cross the plane located at $z$, as illustrated in upper frame of Fig. 2.
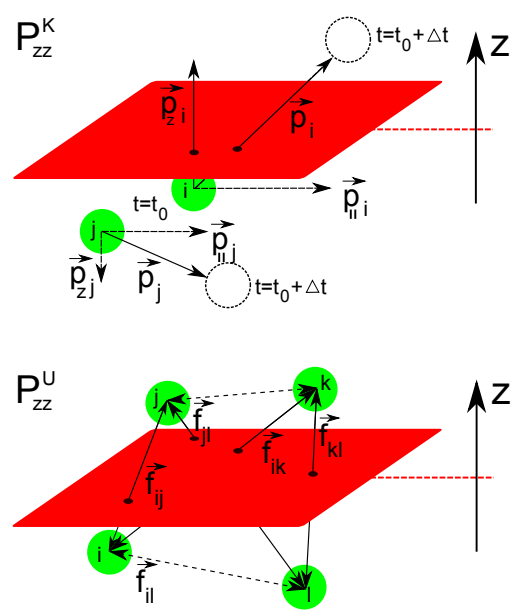

Fig. 2. Illustration of the way the kinetic (upper graph) and potential (lower graph) components of the normal pressure are evaluated with the MOP method in Eqs.(1) and (2). Only the pair interactions marked as the solid arrows contribute to the $P_{z z}^{U}$ and only particles which cross the plane within $d t$ contribute to the $P_{z z}^{K}$

The interaction or configurational part of the pressure tensor zz-component in the MOP description is,

$$
\begin{aligned}
P_{z z}^{U}(z) & =\frac{1}{4 S} \sum_{i} \sum_{j} F_{i j}^{Z}\left[\Theta\left(z_{i}-z\right) \Theta\left(z-z_{j}\right)\right. \\
& \left.-\Theta\left(z_{j}-z\right) \Theta\left(z-z_{i}\right)\right]
\end{aligned}
$$

where $\Theta$ denotes the Heaviside step function and $F_{i j}^{Z}$ is the $\mathrm{z}$-component of the force between particles $i$ and $j$. In the above expression, the role of the products of the Heaviside step function is simply to select the intermolecular force components which cross the plane and to make the $i j$ term not change sign on swapping $i$ with $j$ (see the bottom graph in Fig. 2 for an illustration of this term). Additionally the contribution from the applied external constraints, such as tethering the atoms with springs to sites of an underlying virtual rigid lattice, is

$$
P_{z z}^{T}(z)= \pm \frac{1}{S} \sum_{i=1}^{N} F_{z, i}^{e} \Theta\left(z_{i}-z\right) \Theta\left(z_{m}-z\right),
$$

where $F_{z, i}^{e}$ denotes the $\mathrm{z}$ component of the external force acting on $i$ particle, $z$ is the plane position and $z_{m}$ denotes the position of the middle of the system. The sign of the function in the above equation depends on the side of the system being considered (+ for bottom and - for top). $P_{M O P}$ denotes the value of the sum of all pressure components - the potential, kinetic and constraint part. In what follows, the sum of the potential and kinetic parts is denoted by $P_{C}(z)=P_{z z}^{U}(z)+P_{z z}^{K}(z)$.

The components of MOP given in Eqs. (1), (2) and (3) can be calculated in MD calculations. In this work we explore some technical aspects of their implementation.

\section{SIMULATION DETAILS AND RESULTS}

MD simulations were performed for the atomic system shown in Fig. 1. In the calculations, interactions between all particles were assumed to be of the repulsive pairwise Weeks-Chandler-Anderson (WCA) potential form which has a well-defined cut off at $r_{c u t}=2^{1 / 6} \sigma$, being a shifted Lennard-Jones potential $u(r)=4 \epsilon\left((\sigma / r)^{12}-(\sigma / r)^{6}\right)+\epsilon$ up to this separation [1]. The potential parameters $\sigma$ and $\epsilon$ specify the interaction range and energy, respectively. Additionally particles in the walls (the regions A,C) were tethered to fixed positions or lattice sites with the harmonic potential, $\frac{1}{2} k_{2}\left(\underline{r}_{i}-\underline{r}_{0 i}\right)^{2}$, where $\underline{r}_{0 i}$ is the equilibrium lattice site of atom $i$ and the spring constant was, $k_{2}=57.15$ [19]. It was verified that these values ensured that displacements from equilibrium points of the wall atoms were less than 10 percent of mean distance between the wall particles, which helped to prevent particles diffusing from region $\mathrm{B}$ into regions $\mathrm{A}$ and $\mathrm{C}$. All quantities in this work are in the conventional reduced units i.e., length in $\sigma$, time 
in $\left(m \sigma^{2} / k_{B} T\right)^{0.5}$, energy in $\epsilon$ and atomic mass, $m$, has been set to unity. $k_{B}$ is the Boltzmann's constant. All calculations were for $T^{*}=k_{B} T / \epsilon=1$ and the walls were thermostated with the velocity scaling scheme. Simulations were performed with the number of atoms in each region, $N_{A}=N_{C}=144$ and $N_{B}=252$. In what follows the reduced quantities are without an asterisk. The region B was (initially) a solid constructed out of a face-centered cubic (FCC) lattice (with the [100]-axis along the $\mathrm{x}$-direction). The walls (regions A, C) were amorphous solids or parts of an FCC crystal structure made up of $4 \mathrm{FCC}(100)$ planes $[3 \times 3 \times 4]$ which were commensurate with the FCC structure in the B-region (formed from basic FCC blocks $3 \times 3 \times 7$ ). The equations of motion were integrated with the leapfrog Verlet algorithm using mostly a time step, $d t=0.0005$ for reasons discussed below. To each well equilibrated system at two densities corresponding to the fluid $(\rho=0.8)$ and solid $(\rho=1.1)$ phase the shear was applied. The shear rate was gradually increased and calculations (after an equilibration period) were performed for steady states with $v_{x}=0.0,0.1,0.2,0.3,0.4$ and 0.5 . Averages of quantities of interest were taken from simulations of $2 \times 10^{6}$ time steps duration.

In Fig. 3, an example of the calculated MOP local pressure and all its components are shown. As may be seen, the form of each separate component is fairly structured function of $z$, but which sum up to a constant value, $P_{M O P}$. Note, in the slit the contribution from the external field (springs), $P^{T}$, is negligible and the total pressure $P_{M O P}$, is in practice equal to the sum of the kinetic and interaction terms, $P_{C}=P^{K}+P^{U}$. Also, it is expected that the calculated value of the total pressure $P_{M O P}(z)$ is equal to the normal pressure produced by average total force exerted by the wall on the particles inside the gap i.e., $P_{W}=<F_{z}>/ S$ (and vice versa by Newton's third law).

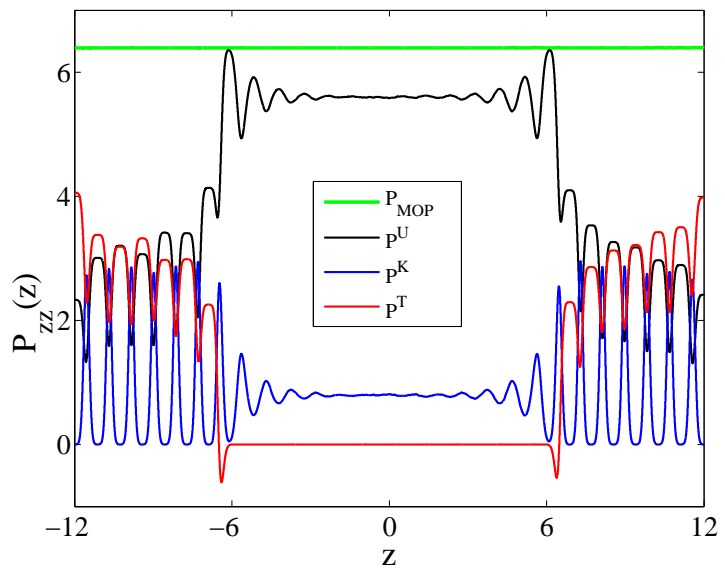

Fig. 3. Illustration of the components in Eqs. (1), (2) and (3) of the local normal pressure, $P_{M O P}(z)$, calculated by the MOP method Calculations are for fluid phase $\left(\rho=0.8, v_{x}=0\right)$ in the slit geometry shown in Fig. 1

There is a potentially important technical point regard- ing the implementation of the MOP expressions. In the MD simulations the equations of motion are integrated with the algorithms which routinely apply a constant time step, $d t$. The potential and kinetic parts of a quantity are calculated at discrete time intervals, $n d t$ where $n=1,2, \ldots$. However, particles cross the plane at discrete times, $t_{i, m}$ which are typically between rather than at integer multiples of $d t$. Localization of the time events $t_{i, m}$ can considerably slow down the calculations. Nevertheless, it might reasonably be expected that the approximation that the plane crossings for the kinetic part be associated with $n d t$ events may become irrelevant on taking a sufficiently small $d t$. This issue is explored in Figs. 4 and 5 which show the difference between the $P_{M O P}$ and the normal pressure value $P_{W}$ for different sizes of the time step, for fluid and solid phase in the slit, respectively. The wall parts of the system are not shown in these two figures.

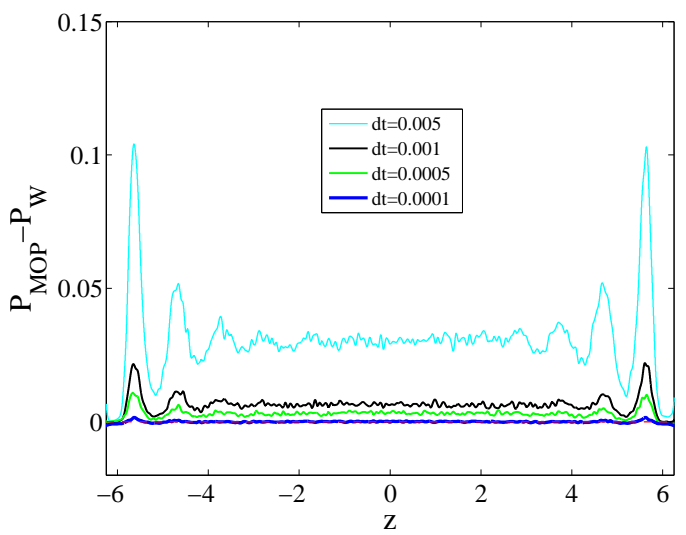

Fig. 4. Difference between the normal pressure calculated by the MOP method for fluid phase $\left(\rho=0.8, v_{x}=0\right)$ in the slit geometry and the normal pressure value $P_{W}$ calculated from the total force exerted by the wall on the system. The solid lines are for different integration time steps used in MD simulations (in each case the long total integration time was the same of $\tau=1000$ ). The accuracy of the lines at any $z$ is about 0.01 . The fluid extends from $z \approx-6$ to 6

The local pressure value should be the same within statistical uncertainly as the calculated value of $P_{W}$. For a fluid central phase, Fig. 4 reveals that for small $d t<0.001$, the difference $P_{M O P}-P_{W}$ tends to zero within the simulation's statistical uncertainty i.e., is less than 0.01 , which means that the sequence of $n d t$ assumed crossings are practically equivalent to the true $t_{i, m}$ set. For $d t=0.001$ the correct flat dependence is obtained for almost the entire slit width but its departure from $P_{W}$ (or a shift from zero) is only marginally within the error bar. The application of larger time steps leads to the shifts which are outside statistical uncertainties. Additionally, as seen for $d t=0.005$, the line is not horizontal but there is some noticeable and unphysical oscillatory structure in the local pressure profile. To summarise, for the type of simulated system and imposed conditions consider in this work an integration time step of order 0.001 is sufficient to obtain statistically consistent results for calculated quantities. Fig. 5 
shows that in the case in which the solid (crystal structure) is in the central B-region, the influence of $d t$ may be more pronounced. In particular a noticeable structure in $P_{M O P}(z)$ (and $P_{C}(z)$ ) persists even for small time steps, $d t<0.001$. In this case only $d t=0.0005$ gives results which are only marginally within the error bar (i.e., 0.05). The oscillations in the local pressure correlate with the oscillations in the density distribution structure, $\rho(z)$ (which are shown in Fig. 7).

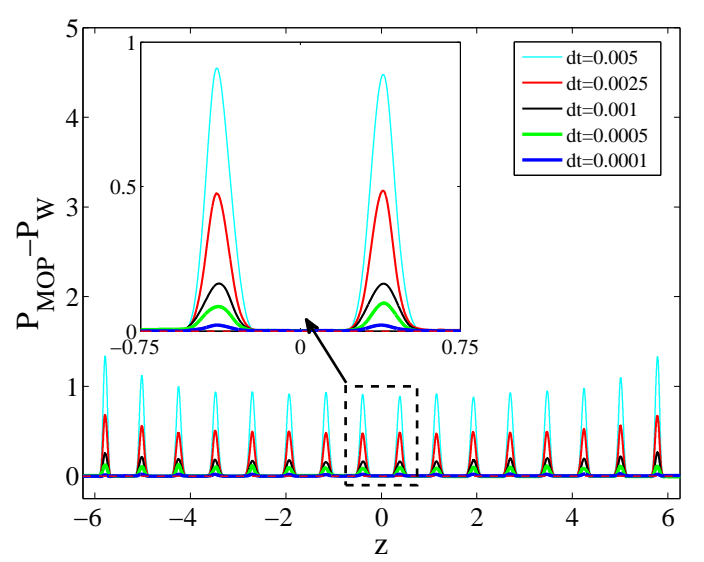

Fig. 5. As for Fig. 4 but the results are for the solid phase in the gap $\left(\rho=1.1, v_{x}=0\right)$. The accuracy of the lines at any $z$ is about 0.05 . The inset shows an enlargement of the central part

The time step tests whose results are shown in Figs. 4 and 5 , indicate that the approximation in the numerical calculation of the kinetic part of the local pressure can be made statistically irrelevant by a reduction of the time step. A further reduction by a factor two to $d t=0.0005$ was used so that we could be confident we had achieved the satisfactory limit in regard to this effect.

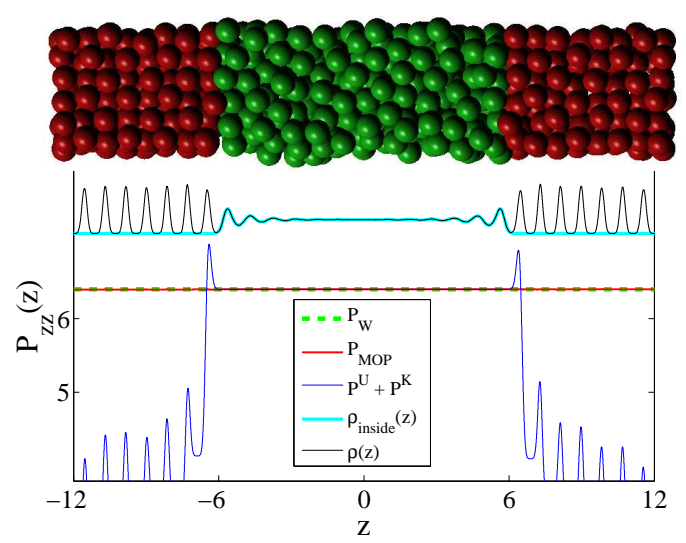

Fig. 6. The normal pressure calculated by the MOP method for $\rho=0.8$ and $v_{x}=0$ marked as the solid line (red). The (dashed green) horizontal line represents the value of the normal pressure calculated as the average total force exerted by the wall on the system, $P_{W}$. The upper solid lines represent the total, $\rho(z)$, and between walls or the inside, $\rho_{\text {inside }}(z)$, particle density distributions across the slit. In the top, a typical configuration of fluid phase between the FCC walls is shown
The calculated normal pressure, $P_{M O P}$, in fluid phase is shown in Fig. 6. In the calculations the inter plane resolution separation was equal to 0.05 . The horizontal line represents the normal pressure value obtained as total z-force per unit area $P_{W}$. Both quantities agree very well anywhere within the liquid. On the figure the liquid is characterized by the density distribution, $\rho_{\text {inside }}(z)$ (middle frame) and illustrated by a snapshot configuration between the FCC walls (top frame). Thus an accurate evaluation of the normal pressure can be achieved through $P_{M O P}$, averaged over an arbitrary number of planes placed anywhere within the slit, which also applies for wall-driven sheared confined fluids.

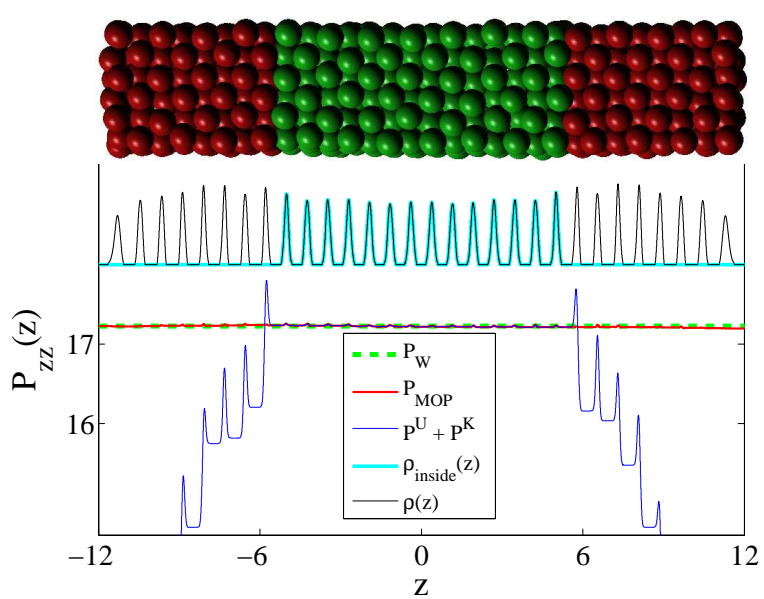

Fig. 7. As for Fig. 6 except that there is a solid phase in the gap $(\rho=1.1)$

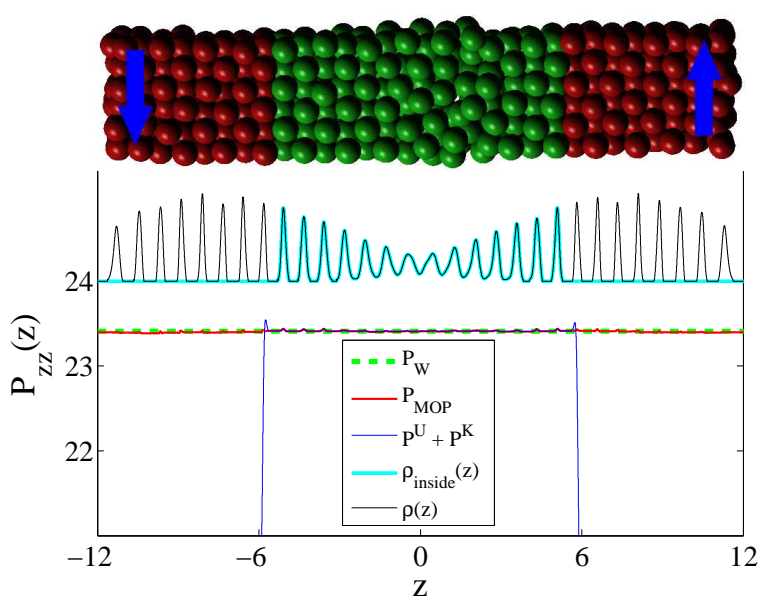

Fig. 8. As for Fig. 7 except that the wall speed was $v_{x}=0.5$. In the slit the formation of the central localization (CL) phase [22] or boundary-driven shear flow is observed. The magnitude of the scillations in the density profile distribution damp out towards the center indicating liquid-like character of the system in this part of the slit

The situation in which there is a solid or partially solid structure between static or movable slit walls is shown in Figs. 7 and 8. Also in this case the expected agreement 
between $P_{M O P}$ and the $P_{W}$ is achieved well within the simulation statistical uncertainty. However, the numerical errors associated with computing the kinetic and configurational parts of MOP can be non-negligible and should be a consideration when determining the algorithm parameters, especially when there is a solid phase in the gap between the walls. In particular, our calculations indicate that attention should be paid in situations in which densely packed structures are investigated for which $\rho(z)$ displays strong oscillations.

\section{CONCLUSIONS}

These calculations confirm that the MOP method is a valuable method for determining the local pressure tensor for highly inhomogeneous particle systems in a slit geometry and steady states conditions at equilibrium as well as away from equilibrium. In our MD simulations, the MOP's kinetic part is evaluated up to the time step resolution. It was found that a reduction of an integration time step to a still practical value is sufficient to give an accurate limiting estimation of this quantity. In numerical calculations using the MOP expressions, a study of the influence of the magnitude of the time step is therefore recommended as a routine preliminary task. Finally, our results suggest that calculation of the instantaneous normal pressure involved in barostat schemes is more conveniently performed with the total force formula than with the MOP scheme.

\section{Acknowledgments}

The work has been partially supported by the National Science Center grant DEC-2012/05/B/ST3/03255.

\section{References}

[1] M.P. Allen and D.J. Tildesley, Computer Simulation of Liquids, Oxford University Press: 1987.

[2] Z. Zhou, Fluctuations and thermodynamics properties of the constant shear strain ensemble, J. Chem. Phys. 114, 87698774 (2001).

[3] H.W. Graben and J.R. Ray, Unified treatment of adiabatic ensembles, Phys. Rev. A 43, 4100-4103 (1991).

[4] J.R. Ray and A. Rahman, Statistical ensembles and molecular dynamics studies of anisotropic solids, J. Chem. Phys. 80, 4423-4428 (1984).

[5] H.C. Andersen, Molecular dynamics simulations at constant pressure and/or temperature, J. Chem. Phys. 72, 2384-2393 (1980).
[6] W.G. Hoover, Constant-pressure equations of motion, Phys. Rev. A 34, 2499-2500 (1986).

[7] J.R. Ray, Pressure fluctuations in statistical physics, Am. J. Phys. 50, 1035 (1982).

[8] M. Parrinello and A. Rahman, Crystal Structure and Pair Potentials: A Molecular-Dynamics Study, Phys. Rev. Lett. 45, 1196-1199 (1980).

[9] M. Parrinello and A. Rahman, Polymorphic transitions in single crystals: A new molecular dynamics method, J. Appl. Phys. 52, 7182-7190 (1981).

[10] E. Hernández, Metric-tensor flexible-cell algorithm for isothermal-isobaric molecular dynamics simulations, J. Chem. Phys. 115, 10282-10290 (2001).

[11] D.J. Evans and G.P. Morriss, Isothermal-isobaric molecular dynamics, Chem. Phys. 77, 63-66 (1983).

[12] H.J.C. Berendsen, J.P.M. Postma, W.F. van Gunsteren, A. DiNola and J.R. Haak, Molecular dynamics with coupling to an external bath, J. Chem. Phys. 81, 3684-3690 (1984).

[13] J.-C. Wang and K.A. Fichthorn, A method for molecular dynamics simulation of confined fluids, J. Chem. Phys. 112, 8252-8259 (2000).

[14] M. Lupkowski and F. van Swol, Computer simulation of fluids interacting with fluctuating walls, J. Chem. Phys. 93, 737-745 (1990).

[15] M. Lupkowski and F. van Swol, Ultrathin films under shear, J. Chem. Phys. 95, 1995 (1991).

[16] H. Eslami, F. Mozaffari, J. Moghadasi and F. MüllerPlathe, Molecular dynamics simulation of confined fluids in isosurface-isothermal-isobaric ensemble, J. Chem. Phys. 129, 194702 (2008).

[17] H. Takaba, Y. Onumata and S.-I. Nakao, Molecular simulation of pressure-driven fluid flow in nanoporous membranes, J. Chem. Phys. 127, 054703 (2007).

[18] J. Cormier, J.M. Rickman and T.J. Delph, Stress calculation in atomistic simulations of perfect and imperfect solids, J. App. Phys. 89, 99-104 (2001).

[19] B.D. Todd, D.J. Evans and P.J. Davis, Pressure tensor for inhomogeneous fluids, Phys. Rev. E 52, 1627-1638 (1995).

[20] D.M. Heyes, E.R. Smith, D. Dini and T.A. Zaki, The equivalence between volume averaging and method of planes definitions of the pressure tensor at a plane, J. Chem. Phys. 135, 24512 (2011).

[21] J. Petravic and P. Harrowell, The boundary fluctuation theory of transport coefficients in the linear-response limit, J. Chem. Phys. 124, 014103 (2006).

[22] D.M. Heyes, E.R. Smith, D. Dini and H.A. Spikes and T.A. Zaki, Pressure dependence of confined liquid behavior subjected to boundary-driven shear, J. Chem. Phys. 136, 134705 (2012).

[23] S. Butler and P. Harrowell, Simulation of the coexistence of a shearing liquid and a strained crystal, J. Chem. Phys. 118, 4115-4126 (2003).

[24] S. Butler and P. Harrowell, Factors determining crystal-liquid coexistence under shear, Nature (London) 415, 1008-1011 (2002).

[25] J.H. Irving and J.G. Kirkwood, The Statistical Mechanical Theory of Transport Processes. IV. The Equations of Hydrodynamics, J. Chem. Phys. 18, 817-829 (1950). 

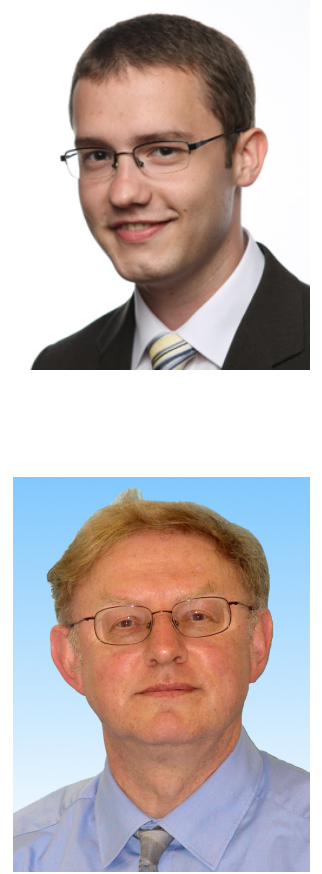

D.M. Heyes obtained a degree in Chemistry from the University of Bristol in 1974. He was awarded a PhD in the area of the molecular dynamics of solids and surfaces from UMIST in 1977, under the supervision of Professors M. Barber and J.H.R. Clarke. After post-doctoral positions at the Catholic University of America, Washington DC, USA, University of Amsterdam, the Netherlands, and then Royal Holloway College University of London, he was a Royal Society 1983 Research Fellow between 1983 to 1992. Since 2008 he has been a member of the Tribology Group in the Department of Mechanical Engineering, Imperial College London. His research interests include Non-equilibrium Molecular Dynamics (NEMD) simulations and associated statistical mechanics applied to tribological systems.

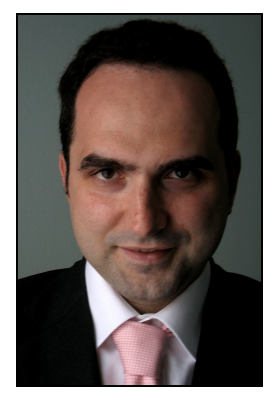

D. Dini, CEng and Member of the UK Institution of Mechanical Engineers (IMechE), Member of the American Society of Mechanical Engineering (ASME), Fellow of the Higher Education Academy, holds a post as a Reader in Tribology at the Department of Mechanical Engineering of Imperial College London. Prior to joining Imperial College, and after receiving a Master in Mechanical Engineering from the Politecnico di Bari (Italy) in 2000, Dr. Dini studied for a D.Phil. in Solid Mechanics at the University of Oxford. In 2004 he has been awarded the Tribology Trust Bronze Medal by the IMechE for his early contribution to the field and, in particular, to the design of fan blades in gas turbine engines. His has since been awarded three best research papers prizes and he is the recipient of a number of national and international honours and awards. $\mathrm{He}$ is Assistant Editor of the International Journal of Solids and Structures and sits on other three editorial boards of international journals.

Dr. Dini's research interests include advanced numerical methods for modelling solids, fluids and fluid/solid interactions, surface characterisation, contact mechanics, fatigue and fretting, fracture, elasto-plastic material modelling and characterisation, X-ray and neutron diffraction, biotribology, nano-tribology and molecular modelling.

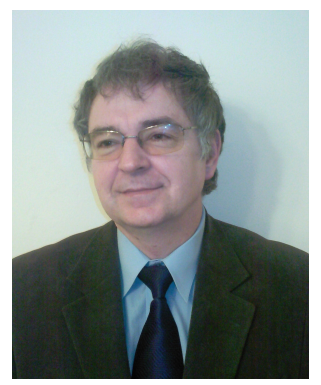

A.C. Brańka, Prof. IFM PAN, studied physics at the Adam Mickiewicz University in Poznań. He obtained his PhD in ISAS in Trieste in 1986 on the subject of computer simulation methods under the supervision of Professor Michele Parrinello. After his PhD he joined the Institute of Molecular Physics Polish Academy of Science, where in 2002 he received habilitation. His interest are in the structural, thermodynamics and transport properties of liquids and suspensions, anomalous elasticity in solids and simulation methods with particles. 